\title{
Gestión del cuidado de enfermería en infección por Acinetobacter Baumanniï: caso clínico
}

Karla Judith Ruíz-González (D) 1; Luis Arturo Pacheco-Pérez (D) 2*;

María de los Ángeles Paz-Morales (D) ${ }^{3}$

\section{RESUMEN}

Introducción: La infección por Acinetobacter Baumannii representa un problema de salud pública a nivel mundial debido a la resistencia a los antibióticos que ha desarrollado la bacteria y los nuevos brotes nosocomiales. Objetivo: Describir la evidencia de un caso clínico que se desarrolló en la Ciudad de Chihuahua, México, presentando resultados positivos al implementarse un plan de cuidados en el paciente y profesionales de enfermería. Metodología: Se realizó una valoración a partir de los patrones funcionales de salud de Marjory Gordon; se empleó una taxonomía de enfermería para elaborar etiquetas diagnósticas dirigidas al paciente y profesionales de la disciplina. Presentación del caso: Se trata de un paciente de 34 años de edad que ingresó a la unidad de cuidados intensivos de un hospital público por accidente automovilístico, posteriormente los cultivos de heridas y catéteres resultaron positivos de para Acinetobacter baumannii. Conclusiones: Las taxonomías resultaron efectivas para estabilizar la salud del paciente cuando estaba comprometido su estado hemodinámico. Se sugiere complementar con resultados de evidencia científica cuando las etiquetas diagnósticas y plan de cuidados sean dirigidos a los profesionales de enfermería.

Palabras clave: Cuidados Críticos; Atención de Enfermería; Infección; Concienciación; Sensibilización Pública (DeCS).

1 'Doctora en Administración. Supervisión de Enfermería, Hospital General "Dr. Salvador Zubirán Anchondo". Chihuahua, Chihuahua, México.

2 Doctor en Ciencias de Enfermería. Universidad de Sonora. Departamento de Ciencias de la Salud, Obregón, Sonora, México.

${ }^{4}$ Doctora en Educación. Universidad Autónoma de Nuevo León. Facultad de Enfermería. Monterrey, Nuevo León, Mexico.

Recibido: 09/01/2020

Aceptado: 25/03/2020

* Autor para correspondencia: luispachecoperez@gmail.com

Copyright $\odot 2020$ SANUS

Artículo de acceso abierto distribuido

bajo Licencia Creative Commons

\section{cc) (i) $\ominus$

\section{Cómo citar este artículo}

Ruíz-González KJ, Pacheco-Pérez LA, Paz-Morales MDLA. Gestión del cuidado de enfermería en infección por Acinetobacter Baumannii: caso clínico. SANUS. 2020; (13):1-10.[Acceso____ ]; Disponible en: 


\title{
Nursing care management in an Acinetobacter Baumannii infection: clinical case
}

\begin{abstract}
Introduction: The infection caused by Acinetobacter Baumannii is a worldwide public health problem due to the resistance developed by the bacteria to antibiotics and new nosocomial outbreaks. Objective: Describe the evidence of a clinical case that took place in the City of Chihuahua, Mexico, presenting positive results when a care plan was implemented on the patient and nursing staff. Methodology: An assessment was carried out based on the Marjory Gordon's functional health patterns; a nursing taxonomy was used to prepare diagnosis labels aimed to the patient and nursing staff. Case presentation: A 34 year-old patient was admitted to the intensive care unit of a public hospital due to a car accident; subsequently, wounds and catheter cultures were positive for Acinetobacter Baumannii. Conclusions: The nursing taxonomies were effective to stabilize the patient's health when his hemodynamic status was compromised. It is suggested to complement with the results of scientific evidence when the diagnosis labels and care plan are addressed to nursing professionals.
\end{abstract}

Key words: Critical care; Nursing care; Infection; Awareness; Public awareness(DeCS). 


\title{
Gestão do cuidado de enfermagem na infecção por Acinetobacter Baumannii: caso clínico
}

\begin{abstract}
ABSTRATO
Introdução: A infecção por Acinetobacter Baumannii representa um problema de saúde pública em todo o mundo devido à resistência aos antibióticos desenvolvidos pela bactéria e a novos surtos nosocomiais. Objetivo: Descrever a evidência de um caso clínico desenvolvido na cidade de Chihuahua, México, apresentando resultados positivos quando implementado no paciente e nos profissionais de enfermagem. Metodologia: Foi realizada uma avaliação com base nos padrões funcionais de saúde de Marjory Gordon; uma taxonomia de emfermagem foi usada para desenvolver rótulos de diagnóstico para pacientes e enfermeiros. Apresentação do caso: Paciente de 34 anos, internado em unidade de terapia intensiva de um hospital público por acidente de carro; posteriormente, as culturas de feridas e cateteres foram positivas para Acinetobacter Baumannii. Conclusões: As taxonomias da enfermagem foram eficazes na estabilização da saúde do paciente quando seu estado hemodinâmico estava comprometido. Sugere-se complementar os resultados de evidências científicas quando os rótulos diagnósticos e o plano de cuidados são endereçados aos profissionais de enfermagem.
\end{abstract}

Palavras chave: Cuidados críticos; Cuidados de enfermagem; Infecção; Conscientização; Sensibilização pública (DeCS). 


\section{INTRODUCCIÓN}

Acinetobacter se refiere a un grupo de bacterias del tipo coco gram-negativo que se encuentran en el agua, tierra y piel; presentan un grado bajo de virulencia pero se manifiestan cada vez más en pacientes hospitalizados, especialmente en la unidad de cuidados intensivos (UCI). La Acinetobacter es capaz de colonizar en heridas sin causar infección; sin embargo, uno de los tipos más frecuentes es la Acinetobacter baumannii (AB) a quien se le atribuye el $80 \%$ de los casos de enfermedades provocadas por este patógeno y se asocia principalmente con neumonía, infecciones del tracto urinario, piel y bacteremia ${ }^{(1,2)}$.

Una de las formas más comunes de transmisión de $A B$ es a través del contacto directo con los pacientes debido a su colonización en las manos de los trabajadores de la salud, además la bacteria se encuentra presente en superficies y equipo médico, incluso se han reportado casos en donde al examinar el aire de los cuartos el cultivo resulta positivo, elevando así el riesgo por exposición ambiental. La evidencia muestra que existe una relación entre la infección por AB y los pacientes sometidos a ventilación mecánica, con catéteres intravenosos o urinarios y personas que han sido expuestas previamente a tratamiento farmacológico con carbapenémicos ${ }^{(2-3)}$.

La infección por AB ha tomado relevancia como problema de salud pública a nivel mundial secundario a la resistencia que la bacteria ha desarrollado a los antibióticos de amplio espectro, incluyendo carbapenémicos. La Organización Mundial de la Salud (OMS) dio a conocer una lista de patógenos que requieren de manera urgente estudios farmacológicos para el desarrollo de nuevos antibióticos, colocando a la $A B$ resistente a carbapenémicos en nivel de prioridad 1 o crítica, de tres niveles posibles; sin embargo, es importante mencionar que se deben realizar estudios clínicos rigurosos a cada paciente debido a la amplia diferencia en cuanto a la resistencia a antibióticos que ha mostrado este grupo de bacterias ${ }^{(3-4)}$.

Debido a los nuevos brotes nosocomiales por AB y su resistencia a los antibióticos, la educación para la salud se presenta como una herramienta eficaz para desarrollar conciencia sobre esta infección. La gestión de los cuidados realizados por el profesional de enfermería en la UCI debe incluir una valoración crítica que tome en cuenta los factores de riesgo mencionados y medidas de asepsia y antisepsia, además de la educación al equipo multidisciplinario de salud y familiares con el fin de coadyuvar en la prevención o diagnóstico oportuno de la infección por AB.

Por lo anterior, se presenta el caso clínico de un paciente con infección por $A B$ que ingresó a la $\mathrm{UCl}$ de un hospital público de segundo nivel en Chihuahua, México; se desarrolló un plan de cuidados basado en una taxonomía enfermera dirigido a estabilizar la salud del paciente que se encontraba en estado crítico al salir de quirófano y otro con la finalidad de brindar educación a los profesionales de enfermería, posterior al resultado positivo para $A B$ y así fomentar la conciencia sobre el riesgo de infección por este patógeno; se incluyen además las acciones realizadas por el equipo multidisciplinario de salud del hospital para evitar futuros brotes.

\section{METODOLOGÍA}

El estudio se fundamentó en las etapas del proceso de enfermería (5); la valoración se realizó a través de los 11 Patrones Funcionales de Marjory Gordon ${ }^{\left({ }^{6}\right)}$, que permitió obtener información referente al estado de salud del paciente. El razonamiento efectuado con base en los dominios, determinó los diagnósticos enfermeros de acuerdo a la taxonomía de la North American Nursing Diagnosis Association (5) (NANDA), posterior a ello se planearon los cuidados de enfermería conforme a la taxonomía Nursing Outcomes Classification (7) (NOC) y Nursing Interventions Classification ${ }^{(8)}$ (NIC), estableciendo los resultados esperados para las intervenciones.

\section{PRESENTACIÓN DEL CASO}

Paciente masculino de 34 años de edad con antecedente de accidente automovilístico, ingresa a la UCI procedente de quirófano, post operado de laparotomía exploradora, fijación de fémur derecho por fractura expuesta y traumatismo craneoencefálico severo. De acuerdo con interrogatorio a familiares el paciente no cuenta con antecedentes personales patológicos, refieren consumo de alcohol y tabaco de manera social. A su ingreso a la UCI se inició tratamiento antibiótico profiláctico con cefalosporina de tercera generación y clindamicina. Sus resultados de laboratorio: $\mathrm{Hb} 10.10 \mathrm{~g} / \mathrm{dL}$, plaquetas 429,000 mm3 y leucocitos 17,000 uL. A los 7 días de estancia en la UCl se realizaron cultivos de catéter, herida y secreción bronquial, resultando positivo para $\mathrm{AB}$.

\section{Valoración por patrones funcionales}

\section{Patrón 1: percepción de la salud}

No valorable.

\section{Patrón 2: nutricional - metabólico}

Presenta sobrepeso, índice de masa corporal de $26,4 \mathrm{~kg} / \mathrm{m} 2$. Se encuentra en ayuno.

\section{Patrón 3: eliminación}

Presenta sonda vesical tipo Foley tamaño 16, sin gasto urinario presente al momento de la valoración. Función 
Tabla 1. Plan de cuidados de enfermería al paciente con infección por Acinetobacter baumanii

\begin{tabular}{|c|c|c|}
\hline NANDA & NOC & NIC \\
\hline $\begin{array}{l}\text { Deterioro del intercam- } \\
\text { bio de gases (00030). } \\
\text { Condición asociada: } \\
\text { Desequilibrio en la } \\
\text { ventilación-perfusión } \\
\text { Características definito- } \\
\text { rias: } \\
\text { Patrón respiratorio } \\
\text { anormal. } \\
\text { Taquicardia }\end{array}$ & $\begin{array}{l}\text { Estado respiratorio: } \\
\text { ventilación. } 0403 \\
\text { Indicadores: } \\
\text { 040315- Ortopnea } \\
\text { Estado respiratorio: } \\
\text { intercambio gaseo- } \\
\text { so; } 0402 \\
\text { Indicadores: } \\
\text { 040211- Sat } 02\end{array}$ & $\begin{array}{l}\text { Manejo de la vía aérea } 3140 \\
\text { Actividades: } \\
\text { Colocar al paciente para maximizar el potencial de ventilación } \\
\text { - Auscultar los sonidos respiratorios, observando las áreas de dismi- } \\
\text { nución o ausencia de ventilación y la presencia de sonidos adventi- } \\
\text { cios } \\
\text { Manejo de la ventilación mecánica: invasiva } 3300 \\
\text { Actividades: } \\
\text { - Controlar las condiciones que indican la necesidad de soporte } \\
\text { ventilatorio } \\
\text { Controlar las actividades que aumentan el consumo de } 02\end{array}$ \\
\hline $\begin{array}{l}\text { Disminución del gasto } \\
\text { cardiaco (00029). } \\
\text { Condición asociada: } \\
\text { Alteración de la poscar- } \\
\text { ga } \\
\text { Características definito- } \\
\text { rias: } \\
\text { Alteración de la presión } \\
\text { arterial } \\
\text { Llenado capilar prolon- } \\
\text { gado }\end{array}$ & $\begin{array}{l}\text { Estado circulatorio: } \\
0401 \\
040101 \text { Presión arte- } \\
\text { rial sistólica } \\
040102 \text { Presión arte- } \\
\text { rial diastólica } \\
\text { 040104 Presión } \\
\text { arterial media }\end{array}$ & $\begin{array}{l}\text { Cuidados cardíacos } 4044 \\
\text { Actividades: } \\
\text { Realizar una valoración exhaustiva de la circulación periférica. } \\
\text { - Monitorizar el estado cardiovascular. } \\
\text { Manejo de líquidos } 4120 \\
\text { - Vigilar el estado de hidratación } \\
\text { Monitorizar el estado hemodinámico, incluidos los niveles de PVC, } \\
\text { PAM, PAP y PECP. }\end{array}$ \\
\hline
\end{tabular}

renal (creatinina, nitrógeno ureico en sangre y urea) dentro de parámetros aceptables, presentando balance hídrico negativo al salir de quirófano.

\section{Patrón 4: actividad-ejercicio}

Apoyo ventilatorio por cánula endotraqueal, campos pulmonares bien ventilados con crepitantes, presenta múltiples lesiones abrasivas en tórax, temperatura corporal de 38.7 oC, frecuencia cardiaca de $123 x^{\prime}$, frecuencia respiratoria de $34 x^{\prime}$, presión arterial de 87/46 mmHg, presión arterial media de 58, dependiente de norepinefrina a $0.4 \mathrm{mcg} / \mathrm{kg} / \mathrm{min}$, herida quirúrgica limpia, con bordes bien afrontados, miembro pélvico derecho con férula, mostrando llenado capilar de 4 segundos. Se encuentra en reposo absoluto.

\section{Patrón 5: sueño-descanso}

Bajo efectos de sedación, con midazolam a $18.5 \mathrm{mcg} / \mathrm{kg} / \mathrm{min}$, fentanilo a $18.5 \mathrm{mcg} / \mathrm{kg} / \mathrm{min}$ y propofol a $27.7 \mathrm{mcg} / \mathrm{kg} / \mathrm{min}$.

\section{Patrón 6: cognitivo-perceptivo}

La escala de la agitación y sedación Richmond de -4, pupilas anisocóricas (izquierda $=2 \mathrm{~mm}$, derecha $=4 \mathrm{~mm}$ ).

\section{Patrón 7: autopercepción - autoconcepto}

No valorable.

\section{Patrón 8: rol-relaciones}

Vive en casa propia, casado con 3 hijos.

\section{Patrón 9: sexualidad - reproducción}

No valorable.

\section{Patrón 10: tolerancia al estrés}

No valorable.

\section{Patrón 11: valores-creencias}

No valorable.

\section{Plan de cuidados de enfermería}

(Tabla 1 y 2). 


\begin{tabular}{|c|c|c|}
\hline $\begin{array}{l}\text { Riesgo de lesión (00035). } \\
\text { Factores de riesgo: } \\
\text { Infección intrahospita- } \\
\text { laria } \\
\text { Exposición a patógenos } \\
\text { Condición asociada } \\
\text { Alteración en el funcio- } \\
\text { namiento } \\
\text { psicomotor } \\
\text { Población en riesgo: } \\
\text { Deterioro de los meca- } \\
\text { nismos } \\
\text { primarios de defensa }\end{array}$ & $\begin{array}{l}\text { Control del riesgo: } \\
1902 \\
\text { Indicadores: } \\
\text { 190202 Controla los } \\
\text { factores de riesgo } \\
\text { ambientales } \\
\text { 190204 Desarro- } \\
\text { lla estrategias de } \\
\text { control del riesgo } \\
\text { efectivas }\end{array}$ & $\begin{array}{l}\text { Aislamiento } 6630 \\
\text { Actividades: } \\
\text { Designar un miembro del personal de enfermería para que se comu- } \\
\text { nique con el paciente y dirija al resto del personal. } \\
\text { Vigilar la temperatura, limpieza y seguridad de la zona de aislamien- } \\
\text { to. } \\
\text { Control de infecciones } 6540 \\
\text { Actividades: } \\
\text { Limpiar el ambiente adecuadamente después de cada uso por parte } \\
\text { de los pacientes. } \\
\text { Aislar a las personas expuestas a enfermedades transmisibles. } \\
\text { Aplicar las precauciones de aislamiento designadas que sean apro- } \\
\text { piadas. }\end{array}$ \\
\hline $\begin{array}{l}\text { Limpieza ineficaz de las } \\
\text { vías aéreas (00031). } \\
\text { Factores relacionados: } \\
\text { Mucosidad excesiva } \\
\text { Características definito- } \\
\text { rias: } \\
\text { Excesiva cantidad de } \\
\text { esputo } \\
\text { Sonidos respiratorios } \\
\text { adventicios } \\
\text { Condición asociada } \\
\text { Vía aérea artificial }\end{array}$ & \begin{tabular}{|l|} 
Estado respiratorio: \\
permeabilidad de \\
las vías respiratorias: \\
0410 \\
Indicadores: \\
041007 Ruidos respi- \\
ratorios patológicos \\
041020 Acumulación \\
de esputos
\end{tabular} & $\begin{array}{l}\text { Manejo de las vías aéreas artificiales } 3180 \\
\text { Actividades: } \\
\text { Aspirar la tráquea, la cavidad oral y, a continuación, la nasofaringe } \\
\text { para eliminar las secreciones por encima del balón del tubo ET con } \\
\text { el fin de disminuir el riesgo de aspiración. } \\
\text { Mantener el inflado del globo del tubo endotraqueal a } 15-20 \text { mm/Hg } \\
\text { durante la ventilación mecánica } \\
\text { Auscultar la presencia de sonidos pulmonares bilaterales } \\
\text { Mantener el cabecero de la cama elevado a } 30-45^{\circ} \\
\text { Comprobar el color, cantidad y consistencia de las secreciones } \\
\text { Realizar fisioterapia torácica }\end{array}$ \\
\hline $\begin{array}{l}\text { Hipertermia (00007). } \\
\text { Factores relacionados: } \\
\text { Deshidratación } \\
\text { Características definito- } \\
\text { rias: } \\
\text { Piel caliente al tacto } \\
\text { Taquicardia } \\
\text { Condición asociada: } \\
\text { Traumatismo }\end{array}$ & $\begin{array}{l}\text { Severidad de la } \\
\text { infección: } 0703 \\
\text { Indicadores: } \\
070307 \text { Fiebre } \\
070335 \text { Coloni- } \\
\text { zación del acceso } \\
\text { vascular }\end{array}$ & $\begin{array}{l}\text { Baño } 1610 \\
\text { Actividades: } \\
\text { Realizar baño con el agua a una temperatura agradable. } \\
\text { Regulación de la temperatura } 3900 \\
\text { Actividades: } \\
\text { Instaurar un dispositivo de monitorización de temperatura central } \\
\text { continua. } \\
\text { Utilizar compresas de hielo o de gel } \\
\text { Ajustar la temperatura ambiental a las necesidades del paciente } \\
\text { Manejo de un dispositivo de acceso venoso central } 4054 \\
\text { Utilizar una técnica aséptica estricta siempre que se manipule el ca- } \\
\text { téter, se acceda a el o se use para administrar medicación, con el fin } \\
\text { de reducir las infecciones sanguíneas relacionadas con el catéter. } \\
\text { Utilizar dispositivos sin aguja para favorecer un sistema cerrado. } \\
\text { Cambiar los sistemas de infusión de líquidos cada } 72 \text { horas. } \\
\text { Inspeccionar el sitio de entrada a diario en busca de eritema, dolor, } \\
\text { sensibilidad dolorosa, calor o tumefacción, pues los dispositivos se } \\
\text { asocian con un mayor riesgo de infección. }\end{array}$ \\
\hline
\end{tabular}

Fuente: NANDA, NOC y NIC, elaboración propia. 
Tabla 2. Plan de cuidados de Enfermería al profesional de enfermería de la UCl

\begin{tabular}{|c|c|c|}
\hline NANDA & NOC & NIC \\
\hline $\begin{array}{l}\text { Conocimientos deficien- } \\
\text { tes (00126). } \\
\text { Factores relacionados: } \\
\text { Información insuficiente } \\
\text { Conocimiento insuficien- } \\
\text { tes sobre los recursos } \\
\text { disponibles } \\
\text { Características definito- } \\
\text { rias: } \\
\text { Conocimiento insuficien- } \\
\text { te Realización inco- } \\
\text { rrecta de las pruebas }\end{array}$ & \begin{tabular}{|l|} 
Conocimiento: control \\
de la infección : 1842 \\
Indicadores: \\
184202 Factores que \\
contribuyen a la trans- \\
misión de la infección \\
180706 Procedimien- \\
tos de control de la \\
infección \\
184207 Importancia \\
de la higiene de las \\
manos \\
184219 Riesgo de \\
resistencia farmaco- \\
lógica \\
\end{tabular} & $\begin{array}{l}\text { Asesoramiento } 5240 \\
\text { Actividades: } \\
\text { Proporcionar información objetiva según sea necesario y según } \\
\text { corresponda. } \\
\text { Revelar aspectos seleccionados de las experiencias propias o } \\
\text { de la personalidad para dar autenticidad y confianza, si resulta } \\
\text { oportuno. } \\
\text { Control de infecciones } 6540 \\
\text { Actividades: } \\
\text { Aplicar las precauciones de aislamiento designadas que sean } \\
\text { apropiadas. } \\
\text { Enseñar al personal de cuidados el lavado de manos apropiado }\end{array}$ \\
\hline $\begin{array}{l}\text { Disposición para mejo- } \\
\text { rar los conocimientos } \\
\text { (00161). } \\
\text { Características definito- } \\
\text { rias: } \\
\text { Manifiesta deseo de me- } \\
\text { jorar en el aprendizaje }\end{array}$ & \begin{tabular}{|l|} 
Creencias sobre la \\
salud: percepción de \\
amenaza: 1704 \\
Indicadores: \\
170401 Percepción de \\
amenaza para la salud \\
170406 Gravedad per- \\
cibida de la enferme- \\
dad o lesión
\end{tabular} & $\begin{array}{l}\text { Desarrollo de un programa } 8700 \\
\text { Actividades: } \\
\text { Identificar las propuestas alternativas para dirigir las necesidades } \\
\text { o problemas. } \\
\text { Evaluar las propuestas alternativas detallando el coste, los recur- } \\
\text { sos necesarios, la viabilidad y las actividades necesarias. } \\
\text { Facilitar el aprendizaje } 5520 \\
\text { Actividades: } \\
\text { Establecer la información en una secuencia lógica. } \\
\text { Diferenciar el contenido «crítico» del contenido «deseable». }\end{array}$ \\
\hline $\begin{array}{l}\text { Planificación ineficaz de } \\
\text { las actividades (00199). } \\
\text { Factores relacionados: } \\
\text { Percepción no realista de } \\
\text { las acontecimientos } \\
\text { Características definito- } \\
\text { rias: } \\
\text { Recursos insuficientes } \\
\text { Temor por la tarea que } \\
\text { se va a realizar. } \\
\text { Ausencia de un plan }\end{array}$ & \begin{tabular}{|l|} 
Cognición: 0900 \\
Indicadores: \\
090009 Procesa la \\
información \\
090011 Toma decisio- \\
nes apropiadas \\
\\
Creencias sobre la \\
salud: capacidad \\
percibida para actuar: \\
1701 \\
Indicadores: \\
170108 Confianza en la \\
capacidad para llevar \\
a cabo una conducta \\
sobre la salud
\end{tabular} & $\begin{array}{l}\text { Apoyo en la toma de decisiones } 5250 \\
\text { Actividades: } \\
\text { Facilitar la toma de decisiones en colaboración. } \\
\text { Utilizar programas informáticos interactivos o ayudas para la deci- } \\
\text { sión basada en internet como complemento al apoyo profesional. } \\
\text { Educación para la salud } 5510 \\
\text { Identificar los factores internos y externos que puedan mejorar o } \\
\text { disminuir la motivación para seguir conductas saludables. } \\
\text { Formular los objetivos del programa de Educación para la salud. } \\
\text { Identificar los recursos (personal, espacio, equipo, dinero, etc.) } \\
\text { necesarios para llevar a cabo el programa. }\end{array}$ \\
\hline
\end{tabular}




\section{Continuación (Tabla 2)...}

\begin{tabular}{|l|l|l|}
\hline Disposición para mejorar & Participación en las & Orientación en el sistema sanitario 7400 \\
la alfabetización en salud & decisiones sobre asis- & Determinar y facilitar la comunicación entre los profesionales \\
(00262). & tencia sanitaria: 1606 & sanitarios y el paciente/familia, si es el caso. \\
Características definito- & Indicadores: & Enseñanza: procedimiento/ tratamiento 5618 \\
rias: & 160603 Busca informa- & Comentar la necesidad de medidas especiales durante el procedi- \\
Expresa el deseo de & ción contrastada & miento/tratamiento, según corresponda. \\
mejorar el conocimiento & 160606 Identifica prio- & Reforzar la información proporcionada por otros miembros del \\
de los & ridades de los resulta- & equipo de cuidados, según corresponda. \\
determinantes actuales & dos sanitarios & Enseñanza: proceso de enfermedad 5602 \\
de la & 160608 Utiliza técnicas & Explicar la fisiopatología de la enfermedad y su relación con la \\
salud en los entorno & de resolución de pro- & anatomía y la fisiología, según cada caso. \\
sociales y físicos & blemas para conseguir & Identificar las etiologías posibles, según corresponda. \\
Expresa deseo de ob- & los resultados desea- & Identificar cambios en el estado físico del paciente. \\
tener & dos & \\
información suficiente & & \\
para & & \\
navegar por el sistema & & \\
de salud & & \\
\hline
\end{tabular}

Fuente: NANDA, NOC y NIC, elaboración propia.

\section{CONCLUSIONES}

El presente caso clínico muestra la evolución de un paciente de 34 años de edad, politraumatizado que ingresó a la $\mathrm{UCl}$ y posteriormente se diagnosticó infección por AB. Se desarrolló y ejecutó un plan de cuidados basado en las taxonomías NANDA, NOC y NIC en donde se enfatizó en mejorar el estado hemodinámico y respiratorio del paciente puesto que estaban comprometidas su vida y función, además de los cuidados a heridas quirúrgicas y catéteres. Es importante mencionar que los pacientes hospitalizados en la $\mathrm{UCl}$, bajo ventilación mecánica y estancia prolongada, constituyen una población en riesgo de desarrollar una patología asociada a $A B(9,10)$.

Debido al aumento en la tasa de infección producida por $A B$ y su difícil manejo, es necesario desarrollar una cultura de cuidados especializados y conciencia sobre la misma; la farmacoterapia como tratamiento único para el manejo de infecciones provocadas por esta bacteria ha fracasado, por lo que la evidencia muestra un conjunto de intervenciones que ha dado resultado: manejo restringido de carbapenémicos, vigilancia de los factores de riesgo en pacientes que ingresan a la $\mathrm{UCl}$, educación continua al equipo multidisciplinario de salud en lavado de manos, seguir las recomendaciones de los protocolos de desinfección ambiental, aislamiento de pacientes en quienes el cultivo resultó positivo para $A B$ y cultivo de mobiliario y equipo médico al egresar el paciente (11-13).

Posterior a la aplicación de los planes de cuidado que se desarrollaron, el paciente recuperó su estado hemodinámico, se utilizó como antibiótico Ceftazidima con Avibactam, mismo que logró disminuir la infección; sin embargo, no en todos los casos se presentan los mismos resultados positivos (14, 15); se siguieron además las recomendaciones de la evidencia en cuanto a medidas de aislamiento, vigilancia y limpieza del área, por lo que se evitó el riesgo de infecciones cruzadas y el paciente fue dado de alta a hospitalización general sin apoyo ventilatorio. Los profesionales de enfermería manifestaron la importancia de la capacitación continua en cuanto a brotes infecciosos y mostraron disponibilidad para adquirir nuevos conocimientos, además se capacitó en manejo de tecnologías de información y comunicación para la búsqueda de la mejor evidencia.

Pasando a otro orden de ideas, es necesario que al desarrollar planes de cuidado se maneje un lenguaje estandarizado en el que se describan las respuestas humanas, resultados esperados e intervenciones basadas en evidencia científica, tomando en cuenta la valoración completa del paciente y el juicio clínico de los profesionales de enfermería ${ }^{(6)}$. Las taxonomías NANDA, NOC y NIC se adecúan a las necesidades del paciente y su familia; sin embargo, al desarrollar intervenciones sobre conciencia de la enfermedad y educación para la salud dirigidas al personal de enfermería, las taxonomías como método único resultan insuficientes, por lo que se sugiere complementar con hallazgos de estudios científicos y práctica basada en evidencia que se adapte a las necesidades de los profesionales de enfermería y su contexto.

La infección por AB requiere de un conjunto de intervenciones en las que participe el equipo multidisciplinario de 
salud para evitar infecciones cruzadas y disminuir el riesgo de nuevos brotes; el profesional de enfermería desempeña un rol importante al participar en la educación para la salud. Se sugiere complementar las taxonomías NANDA, NOC, NIC con resultados científicos actualizados y adaptarlas al propio contexto cuando se trate de intervenciones dirigidas a los profesionales de enfermería, debido a que la evidencia que se encuentra disponible sobre la efectividad de esta taxonomía en investigación es poca ${ }^{(16)}$.

\section{CONFLICTO DE INTERESES}

Los autores declaran no tener conflicto de intereses.

\section{FINANCIAMIENTO}

No se contó con financiamiento externo.

\section{REFERENCIAS BIBLIOGRÁFICAS}

1. Centers for Disease Control and prevention. Acinetobacter in Healthcare Settings [Internet]; consultado Julio 2019]. Disponible: https://www.cdc.gov/hai/organisms/acinetobacter.html

2. Wong D, Nielsen T, Bonomo R, Pantapalangkoor P, Luna B, Spellberg B. Clinical and Pathophysiological Overview of Acinetobacter Infections: a Century of Challenges.

Clin Microbiol Rev[Internet]. 2017 [consultado Junio 2019];30(1)409-447. Disponible en: http://dx.doi.org/ 10.1128/ CMR.00058-16.

3. Xie R, Zhang X, Zhao Q, Peng B, Zheng J. Analysis of global prevalence of antibiotic resistance in Acinetobacter baumannii infections disclosed a faster increase in OECD countries. Emerg Microbes Infect [Internet]. 2018 [consultado Junio 2019];7(1)31. Disponible en: http://dx.doi.org/ 10.1038/s41426-018-0038-9.

4. World Health Organization (WHO). WHO publishes list of bacteria for which new antibiotics are urgently needed. [Internet]. WHO. 2017 [actualizado Febrero 2017; consultado Julio 2019]. Disponible en: https://www.who.int/news-room/detaiI/27-02-2017-who-publishes-list-of-bacteria-for-which-newantibiotics-are-urgently-needed.

5. Herdman H, Kamitsuru S. NANDA. Diagnósticos enfermeros. Definiciones y clasificación 2018-2020. Edición hispanoamericana. 11 ed. España: Elsevier. 2018.

6. Gordon M. Manual de diagnósticos de enfermería. 11 ed. México D.F. McGraw-Hill Interamericana de España 2007.
7.Moorhead S, Johnson M, Swanson E. Clasificación de resultados de enfermería (NOC). 5th ed. Barcelona España: Elsevier; 2014.

8. Bulecheck G, Butcher H, Dochterman J, Wagner C. Clasificación de intervenciones de enfermería (NIC). 6th ed. Barcelona España: Elsevier España; 2014.

9. Thorne A, Luo T, Dujairajan N, Kaye K, Foxman B. Risk factors for endemic Acinetobacter Baumannii colonization: A case-case study. Chin Med J [Internet]. 2019 [consultado Julio 2019];47(11)1294-1297. Disponible en: https://doi.org/10.1016/j.ajic.2019.04.179.

10. Gao L, Lyu Y, Li Y. Trends in Drug Resistance of Acinetobacter baumannii over a 10-year Period: Nationwide Data from the China Surveillance of Antimicrobial Resistance Program [Internet]. 2017 [consultado Julio 2019];130(6)659664. Disponible en: http://dx.doi.org/ 10.4103/03666999.201601.

11. Cheon S, Kim M, Yun S, Moon J, Kim Y. Controlling endemic multidrug-resistant Acinetobacter baumannii in Intensive Care Units using antimicrobial stewardship and infection control. Korean J Intern Med. [Internet]. 2016 [consultado Julio 2019];31(2)367-374. Disponible en: http:// dx.doi.org/ 10.3904/kjim.2015.178.

12. Hong J, Jang $O$, Bak M, Baek E, Park K, Hong S, Cho $\mathrm{O}$, Bae I. Management of carbapenem-resistant Acinetobacter baumannii epidemic in an intensive care unit using multifaceted intervention strategy. Korean J Intern Med [Internet]. 2018 [consultado Julio 2019];33(5)1000-1007. Disponible en: http://dx. 10.3904/kjim.2016.323.

13. Valencia-Martín R, Gonzalez-Galan V, Alvarez-Marín R, Cazalla-Foncueva1 A, Aldabó T, Gil-Navarro M. Alonso-Araujo I, Martin C, Gordon R, García-Nuñez J, Perez R, Peñalva G, Aznar J, Conde M, Cisneros J. A multimodal intervention program to control a long-term Acinetobacter baumannii endemic in a tertiary care hospital. BMC. [Internet]. 2019 [consultado Diciembre 2019]; 8(199). Disponible en: https://doi.org/10.1186/s13756-019-0658-4

14. Sims S, Neuner E, Benomo A. Ceftazidime-avibactam: a novel cephalosporin/ $\beta$-lactamase inhibitor. Pharm J. [Internet]. 2017 [consultado Diciembre 2019]; 9(5):1-21. Disponible en: http://dx.doi.org/ 10.1211/CP.2017.20202413.

15. Kara E, Yilmaz M, Özbek Çelika B. In vitro activities of ceftazidime/avibactam alone or in combination with antibiotics against multidrug-resistant Acinetobacter bauman- 
nii isolates. J Glob Antimicrob Resist. [Internet]. 2019 [consultado Diciembre 2019]; 17: 137-141. Disponible en: https:// doi.org/10.1016/j.jgar.2018.12.004.

16. Othman E, Shatnawi F, Alrajabi O, Alshraideh J. Reporting Nursing Interventions Classification and Nursing Outcomes Classification in Nursing Research: A Systematic Review. Int J Nurs Knowl [Internet]. 2020 [consultado Enero 2020]; 31(1): 19-36. Disponible en: https://doi.org/10.1111/20473095.12265. 